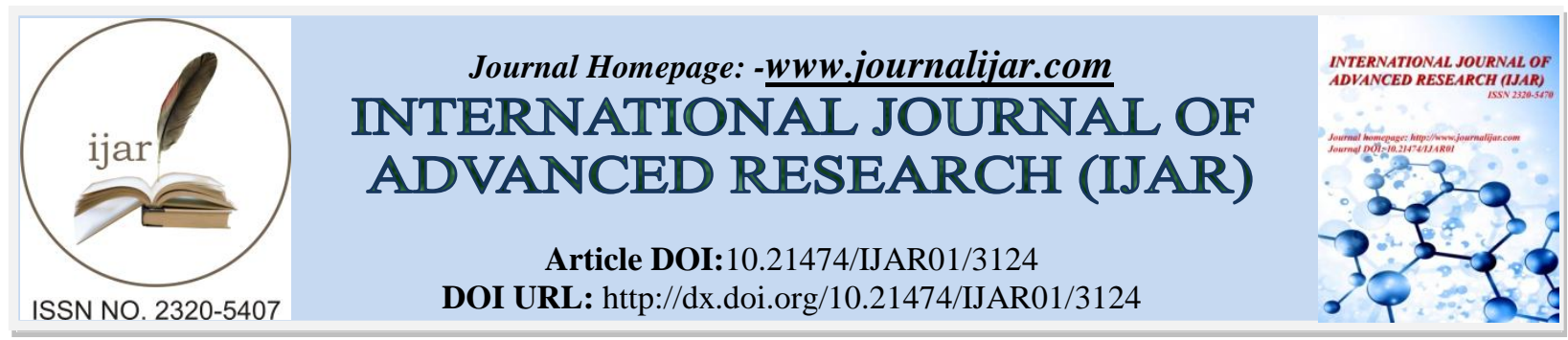

RESEARCH ARTICLE

\title{
LA BRULURE ET LE DIABETE : CORRELATION PHYSIOPATHOLOGIQUE ET THERAPEUTIQUE.
}

A. Khales, A.Arrob, A.N'diaye, F Fouadi, Y.Ribag, M Atmani,K.Ababou, K.Elkhatib And S.Siah. Service de chirurgieplastique, réparatriceet des brûlés, HôpitalMilitaired'Instruction Mohammed V Rabat Maroc.

\section{Manuscript Info}

Manuscript History

Received: 04 December 2016

Final Accepted: 10 January 2017

Published: February 2017

\begin{abstract}
La brûlure chez le patient diabétiqueconstitueun motif fréquentd'admission au service de chirurgieplastique et des brûlés de l'Hôpitalmilitaired'Instruction Mohammed V de Rabat.

Nous rapportonsunesérie de 11 cas de brûluresurvenu chez des patients diabétiques en un an. Les complications dégénératives du diabètesontmises en cause directementà lasurvenue de la brûlure chez six cas. Le délaimoyen de consultation est de septjours. Les brûluressiègent aux membresinférieurs chez neufscas. Quatrepatientsfurentadmis en décompensationacido-cétosique.

La gravité de la brûlureest due à son caractèreprofond sous estimé, par le patient et son entourage, cela a pour conséquence un retard à la consultation et donc de la prise en charge en milieu spécialisé. En fait la brûluresiège le plus souvent aux extrémitésinférieures. Les lésionsdégénératives dues au diabètesontresponsables de la survenue de la brûlure et de sagravité.

La neuropathiediabétiqueestsouventmise en cause dans la survenue de la brûlure et du retard de consultation. Cela rend impératifuneprise en charge thérapeutiquepluridisciplinaireimpliquantchirurgiensplasticiens, réanimateursetendocrinologues.
\end{abstract}

Copy Right, IJAR, 2017,. All rights reserved.

\section{Introduction:}

Les patients diabétiques constituent une population à risqueélevé de brûlure grave, de ce fait le diabètedevrait faire parti des scores de gravités de la brulure.

La neuropathiediabétiqueestune complication fréquente du diabète, et son incidence augmente avec l'âge. La formeclinique la plus fréquenteest la polynévrite «en chaussette» avec perte de la sensibilité nociceptive des pieds et de toute la microcirculation. Elle doitêtrerecherchéesystématiquement par l'examenclinique ${ }^{1}$.

Sa prévalenceestestimée à 50\% chez les diabétiquesdont la maladieévoluedepuis plus de 20 ans, et également à $50 \%$ chez les diabétiquesâgés de plus de 65 ans $^{2}$.

${ }^{1}$ «Diabetes does not influence selected clinical outcomes in critically ill burn patients », (PMID: 21228710), Dahagam CK, Mora A, Wolf SE, Wade CE. J Graver Ressoins [2011]

${ }^{2}$ "The lived experience of a foot burn injury from the perspective of seven Jordanians with diabetes: a hermeneutic Corresponding Author:-A. Khales.

Address:-Service de chirurgieplastique, réparatrice et des brûlés, HôpitalMilitaired'Instruction Mohammed V Rabat Maroc. 
De ce fait, ces troubles sensitifsexpliquent le retard de consultation de cette population de brûlés qui sous estime la gravité de la brûlureet chez qui la lésionestsouvent plus profonde et plus délétère.

Ce travail a pour but de rappelerl'intérêt de la prévention et de la sensibilisation de la population diabétique aux dangers de la brûlure, ainsique demettre en évidence la corrélation entre le diabète et les circonstances de survenu de la brûlure, son évolution et la difficulté de saprise en charge thérapeutique.

\section{Patients etrésultats:}

Nous rapportonsunesérie de 11 cas de brûluresurvenu chez des patients diabétiques en douzemois du 01 janvier 2016 au 31 décembre 2016.

Nous avonsinclusdansce travail les brûluresdomestiquesousurvenusdans un contexted'automédication de trouble neurosensorielliés au diabète, surface cutanéebrûlésinférieur à $20 \%$.

Les complications dégénératives du diabètesontmises en cause directementà lasurvenue de la brûlure chez six cas. Le délaimoyen de consultation est de septjours. Les brûluressiègent aux membresinférieurs chez neufscas. Quatrepatientsfurentadmis en décompensationacido-cétosique.

Après prise en charge médicale des patients et correction de leurs troubles hydro électrolytique et métabolique. Le traitement chirurgical a consisté en une excision des placards de nécrose. Cinq de nos patients onteurecours à unegreffe de peau ( $75 \%$ semi-épaisse; $25 \%$ peautotale) tandisque les deuxautresontbienévolué sous pansementquotidien.

L'oxygénothérapiehyperbare a étéindiquée chez tous les patients ainsiqu'uneantibiothérapieguidée par les données de l'antibiogramme (prélèvementsbactériologiquessystémiquesdevanttoutebrûlure).

Tous les patientsontétéadressés en endocrinologie à leur sortie du service pour uneprise en charge adéquate du diabète.

\section{Discussion:}

La gravité de la brûlureest due son caractèreprofond sous estimé par le patient et son entourage, cela a pour conséquence un retard à la consultation et donc de la prise en charge en milieu spécialisé.En fait la brûluresiège le plus souvent aux extrémitésinférieures. Les lésionsdégénérativesdues au diabètesontsouvent des facteursaggravant la survenue de la brûlureet son évolution ${ }^{3}$.

La brûlure chez les diabétiquesprésente des caractéristiquesdifférentes de la population non diabétique et que la fréquence de cesbrûluresaugmente chez cette population. Ainsi, les patients diabétiquessonthabituellement des patients susceptibles de faire des complications, à cause de la forte présence des dommagesvasculaires de la microcirculation surtout chez les patients indisciplinés, ces troubles affectent des organesmultiples : rein, rétine, nerfspériphériques et peau. Aussi, le diabèteestbienconnu pour êtreassocié à unecapacité curative diminuée et à unesusceptibilité accrue à l'infection, surtout chez les patients mal suivis et qui ontuneglycémie à jeuntrèsélevés. Les polyneuropathies diabétiquessonttrèsfréquentes.Il s'agit le plus souvent de polyneuropathies sensitives. Leurtopographieesthabituellementdistale, bilatérale et symétrique, le plus souvent «en chaussette», plus rarement en gant, et exceptionnellementthoracoabdominale ${ }^{4}$.

${ }^{3}$ "Aging and the pathogenic response to burn» (PMID: 22724078) Rani M, Schwacha MG. Dis vieillissement [2012]

${ }^{4}$ "Clinical outcomes of isolated lower extremity or foot burns in diabetic versus non-diabetic patients: a 10-year retrospective analysis. »(PMID: 22789396) Kimball Z, Patil S, Mansour H, Marano MA, Petrone SJ, Chamberlain RS. Burns , [2013] 
Les manifestations subjectivessont de deuxordres ${ }^{5}$ :

- lesdouleurs: fréquentes, volontiersexacerbées la nuit;

- plussouvent, ils'agit de paresthésies et de dysesthésies (fourmillements, démangeaisons, sensation de froidou de chaud).

D'ailleurs la bruluresurvientsouvent suite à cessymptômes le patient essaye par une source de chaleur :bouillote, barbecue traditionnelouradiateurélectrique de «traiter » sesparesthésies et celaprovoquesouvent la brulure.

L'étude de la brûlure chez les patients diabétiquespermet de constaterque la duréed'hospitalisationet de cicatrisationestnettementsupérieure par rapport à la population non diabétique, avec un risqueaccrud'infection et un grand nombred'interventionschirurgicales à types de paragesitératifs et puis de couverture par greffe de peau.

La prise en charge de ce type de brûlurenécessiteavant tout l'amélioration de la qualité de prise en charge thérapeutique du diabète. La prévention de ce type de traumatismeest un élément indispensable de cetteprise en charge.

Ce travail soulignel'importance des programmesd'éducation de la population diabétiqueafin de diminuer la mortalitéet la morbiditéainsique le coût et le séjour en milieu hospitalier.

Le patient diabétiquesubissantunebrûlure a besoind'une attention particulièreetd'une surveillance plus importante à la recherche des signesd'infections et de décompensation du diabète.

La prise en charge par l'endocrinologuedoit se faire des l'admission du diabétiquebrulés pour prendre en charge le déséquilibreglycémiqueantérieur à la brulureetanticipésurunéventuel trouble glycémiquesurvenant à la suite de la brulure.

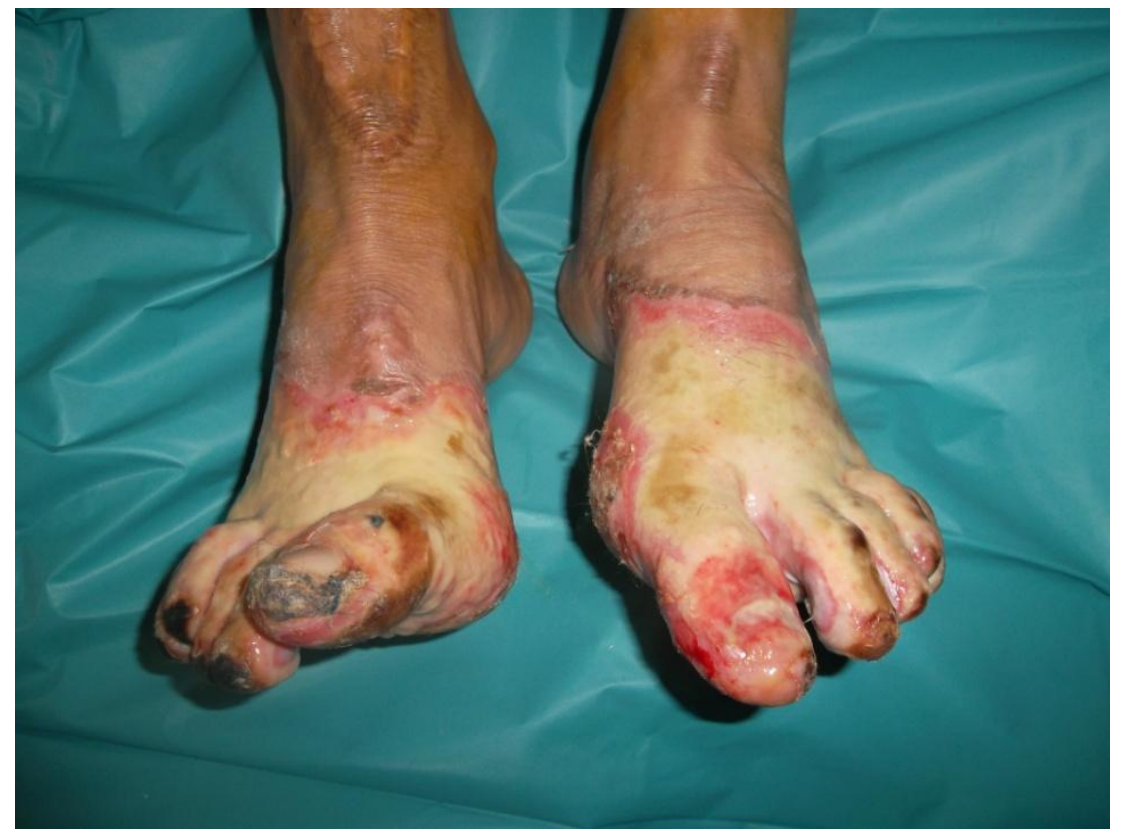

Figure 1:Patient de 65ans brulure par eau bouillante des avantpieds par insensibilitéliée au diabète

${ }{ }_{4}$ Systematic review of complications and outcomes of diabetic patients with burn trauma » (PMID: 27595452) AA Sayampanathan. Burns , [2016] 


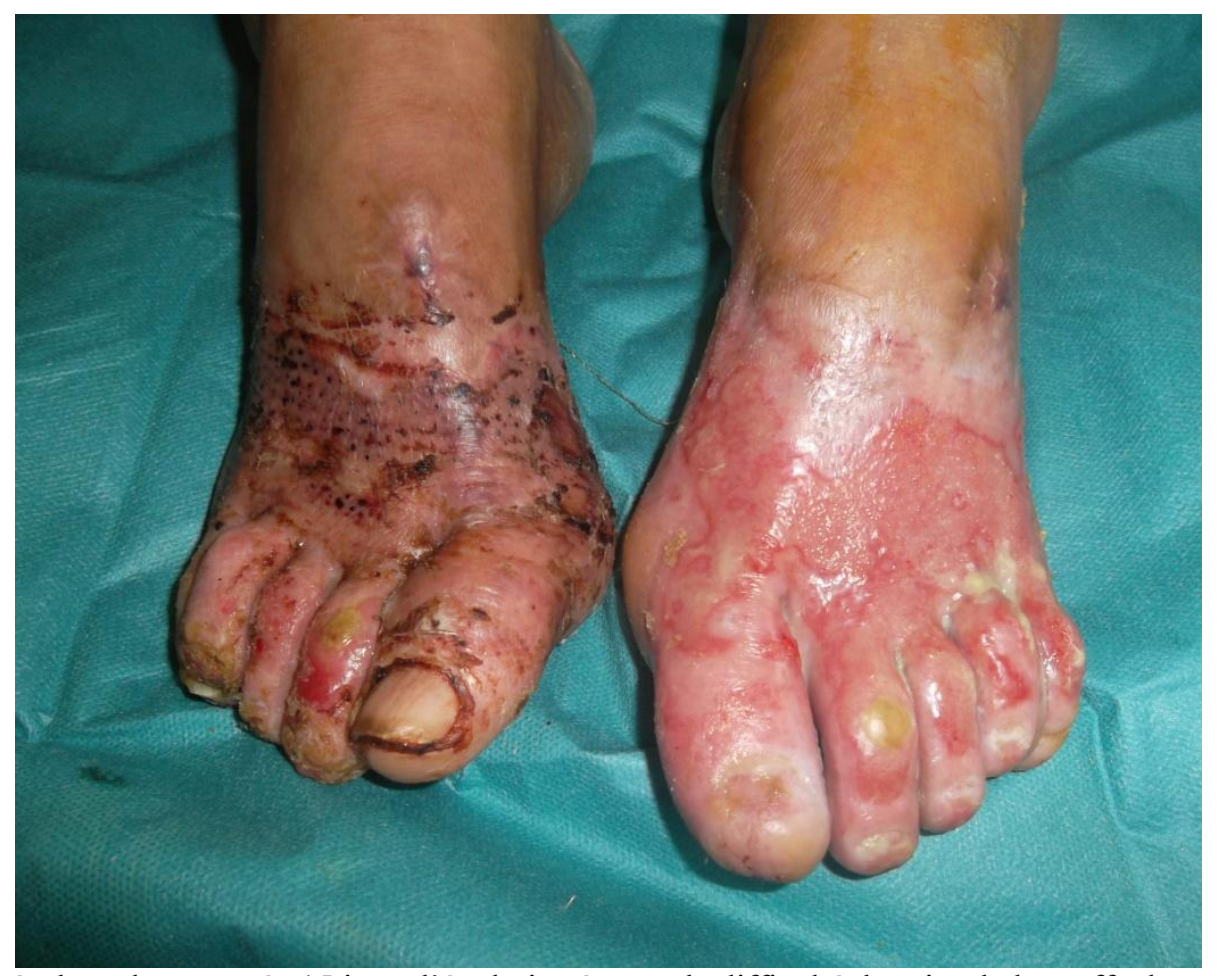

Figure 2:Résultatsobtenu après 15 joursd'évolution à noter la difficulté de prise de la greffe de peau chez les patientsdiabétique

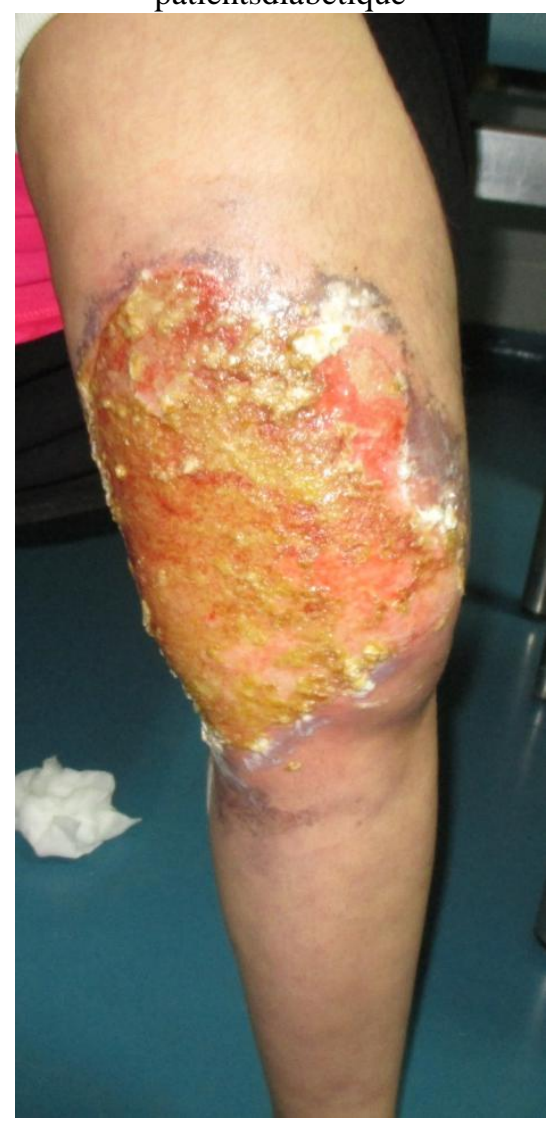

Figure 3: Patiente de 45 ans, brulure thermique par liquide chaud de la cuisse brulure aggravée par le diabete 


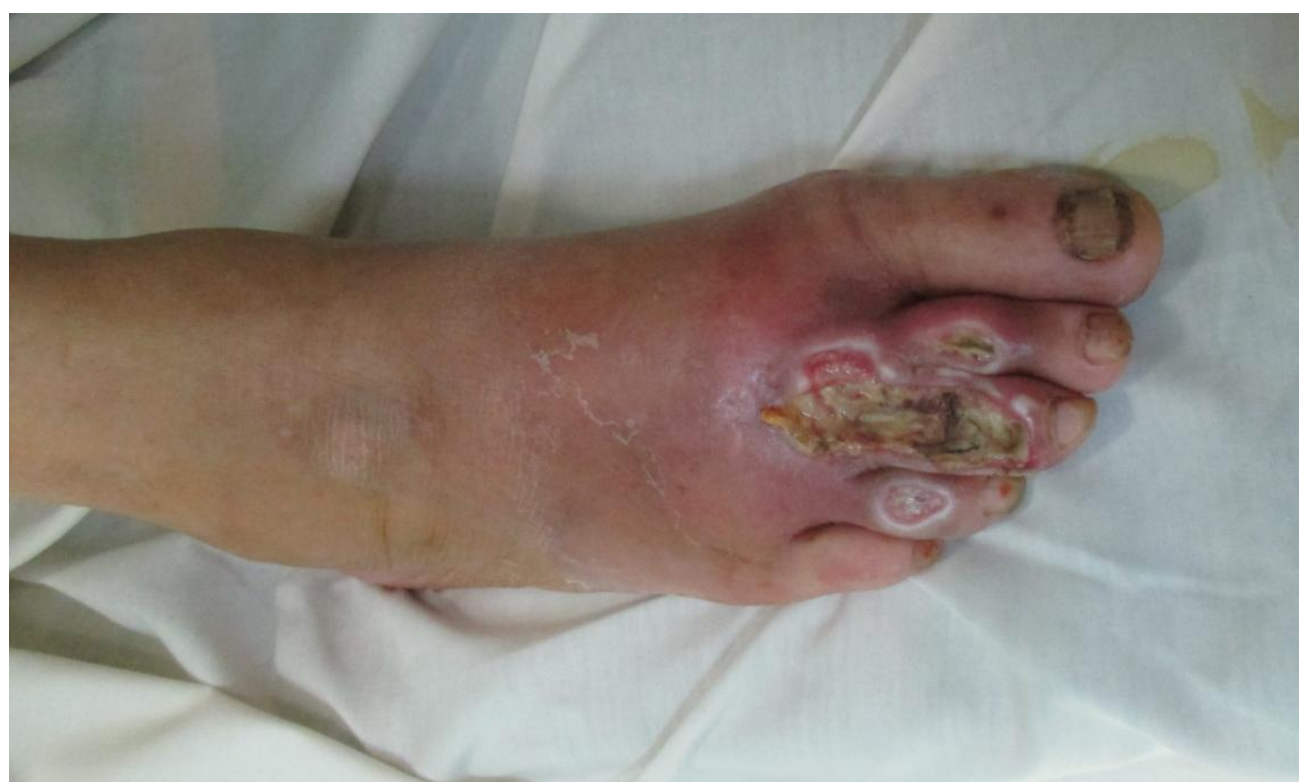

Figure 4:Patient de 53ans brulure du pied survenu suite à un trouble sensitif de la neuropathiediabétique

La neuropathiediabétiqueestune complication fréquente du diabète. La formeclinique la plus fréquenteest la polynévrite «en chaussette» avec perte de la sensibilité des pieds à la douleur. Les brûlures par les bouillottessontl'apanage des patients diabétiquesatteintsd'unepolyneuropathie sensitive, etellessontprofondes. Leurprise en charge thérapeutiqueestdifficile et doitêtremultidisciplinaire. Nous soulignons à traversce travail l'intérêt de l'éducation des patients diabétiques et leursensibilisation aux brûluresdistalesparticulièrement et à la plaiecutanée de façon plus générale.

\section{Conclusion:}

La neuropathiediabétiqueestsouventmise en cause dans la survenue de la brûlure et du retard de consultation. Cela rend impératifuneprise en charge thérapeutiquepluridisciplinaireimpliquantchirurgiensplasticiens, réanimateurset endocrinologues.

\section{References:}

1. «Diabetes does not influence selected clinical outcomes in critically ill burn patients », (PMID: 21228710), Dahagam CK, Mora A, Wolf SE, Wade CE. J Graver Ressoins [2011]

2. "The lived experience of a foot burn injury from the perspective of seven Jordanians with diabetes: a hermeneutic phenomenological study » (PMID: 22051201) Abu-Qamar MZ, Wilson A. Int Wound J [2012]

3. "Aging and the pathogenic response to burn» (PMID: 22724078) Rani M, Schwacha MG. Dis vieillissement [2012]

4. "Clinical outcomes of isolated lower extremity or foot burns in diabetic versus non-diabetic patients: a 10-year retrospective analysis. » (PMID: 22789396) Kimball Z, Patil S, Mansour H, Marano MA, Petrone SJ, Chamberlain RS. Burns , [2013]

5. "Systematic review of complications and outcomes of diabetic patients with burn trauma»(PMID: 27595452) AA Sayampanathan. Burns , [2016]. 